\title{
Positron production in crossed beams of bare uranium nuclei
}

\author{
U. Müller, ${ }^{*}$ T. de Reus, ${ }^{*}$ J. Reinhardt, B. Müller, and W. Greiner \\ Institut für Theoretische Physik der Johann Wolfgang Goethe-Universität Frankfurt, Robert-Mayer-Strasse 8-10, \\ Postfach 111 932, D-6000 Frankfurt am Main 11, Federal Republic of Germany \\ G. Soff \\ Gesellschaft für Schwerionenforschung (GSI), Planckstrasse 1, Postfach 110552, \\ D-6100 Darmstadt 11, Federal Republic of Germany
}

(Received 23 June 1987)

\begin{abstract}
Positron creation in crossed-beam collisions of high-energy, fully stripped heavy ions is investigated within the coupled-channel formalism. In comparison with fixed-target collisions of highly stripped heavy-ion projectiles positron production probabilities are enhanced by more than one order of magnitude. The increase results from the possibility to excite electrons from the negative energy continuum into all bound states. The positron spectrum is shifted towards higher energies because of the absence of electron screening. Rutherford scattering as well as nuclear collisions with time delay are investigated. We also discuss the filling of empty bound states by electrons from pair-production processes.
\end{abstract}

\section{INTRODUCTION}

The process of positron production in heavy-ion collisions has stimulated considerable interest since it is intimately connected to the theory of QED of strong fields. ${ }^{1}$ The combined electric charge $Z_{u}=Z_{P}+Z_{T}$ of two nuclei (projectile and target) brought closely together generates a strong electromagnetic potential which acts on the atomic electrons or, more correctly, on the electronpositron field. Theory predicts that the relativistic Dirac wave functions will be massively distorted by this potential if $Z_{u} \gtrsim 137$, while simultaneously the binding energy of inner-shell states grow strongly. In collisions of two very heavy ions (e.g., $U+U$ ) the combined charge $Z_{u}$ can be easily chosen to reach this region. Then the shrinking of the wave functions has immediate experimental consequences. Theory predicts the excitation of inner-shell holes, $\delta$ electrons, and positrons induced by the rapid variation of the electronic states due to the collision dynamics. These processes have been studied in several experiments ${ }^{2-5}$ and, in their gross properties, are well understood by theory if the essential relativistic effects are properly taken into account.

In this paper we will study the modification of these processes under conditions which up to now have not been realized in experiment. In the future it will be possible to investigate positron production in collisions of two fully ionized atoms. Such collisions of bare nuclei, e.g., uranium on uranium, can be accomplished experimentally using crossed beams of high-energy, fully stripped heavy ions moving in parallel direction with an adjustable intersection angle and thus an adjustable (small) relative velocity. This was discussed previously by Schmelzer and Greiner (see Ref. 6) and now in context with the Schwerionen-Synchrotron (SIS) project at Gesellschaft für Schwerionenforschung, Darmstadt. ${ }^{7}$ In this paper we will discuss the qualitative, and quantitative difference between atomic positron production in fixed-targetscattering experiments and in crossed-beam experiments of bare nuclei, respectively. The formalism describing positron production in heavy-ion collisions as well as qualitative considerations are presented in Sec. II.

The results of numerical calculations presented in Sec. III A demonstrate that, for bare nuclei, pair creation will be enhanced by more than an order of magnitude due to the fact that the inner-shell states, in particular the $K$ shell, are available as a final state for the electron. Section IV demonstrates that as a consequence the quasimolecular $K$ shell will be filled by "capture of electrons from the vacuum" with a probability of about $10^{-2}$.

One important theoretical prediction has been found to be difficult to check: In a static superheavy atom with a charge value $Z_{u}>Z_{\mathrm{cr}} \simeq 173$, the binding energy of the $1 s$ state is expected to exceed the threshold for $e^{+} e^{-}$pair creation. This is predicted to lead to a change of the QED vacuum, signaled by the spontaneous emission of positrons. In this process an electron gets caught in the is state (provided that $K$ holes are present) while a positron is emitted as a free particle. Correspondingly, in a "supercritical" heavy-ion collision the quasimolecular $K$ shell enters the negative-energy continuum as a resonance for internuclear separations of $R<R_{\mathrm{cr}}$, where $R_{\mathrm{cr}}$ depends on the combined charge $Z_{u}$. However, even for the heaviest colliding system investigated up to now, $\mathrm{U}+\mathrm{Cm}$ with $Z_{u}=188$, supercriticality (binding energy $E_{1 s \sigma}^{B}>2 m_{e} c^{2}$ ) lasts only for about $2 \times 10^{-21} \mathrm{~s}$, whereas the spontaneous decay has a much longer characteristic time scale of $\simeq 10^{-19} \mathrm{~s}$. Furthermore, dynamical pair creation due to the nuclear motion which is present also in the subcritical regime becomes sizable. In Ref. 8 a formalism has been developed which includes both "induced" and "spontaneous" positron creation. However, both parts have to be added up coherently to get measurable quantities and the latter contribution under ordinary 
circumstances does not lead to characteristic deviations. Consequently, the spontaneous particle-creation process remains experimentally undetectable in elastic Rutherford scattering.

To overcome this obstacle, it was demonstrated ${ }^{9,10}$ how nuclear reactions leading to a prolonged supercritical field configuration could improve the situation. In the following, we want to retain the formalism of the semiclassical $^{11}$ schematic trajectory model, which has been described in Refs. 10 and 12 and which is briefly sketched in Sec. II. These considerations have led to the following result: During a time-delayed nuclear reaction, the spontaneous positron creation process is systematically enhanced while the induced mechanism only will lead to interference effects. If the delay is a few times $10^{-21} \mathrm{~s}$, both these mechanisms lead to a narrowing of the shape of the positron spectrum. ${ }^{13}$ Such effects have been observed experimentally in the $\delta$-electron and positron spectra of deep-inelastic heavy-ion collisions. ${ }^{14}$ In Sec. III $B$ we discuss how these time-delay effects are modified in collisions of bare nuclei.

If the achievable time delay were considerably larger ( $\gtrsim 10^{-20} \mathrm{~s}$ ), a line due to spontaneous positron emission would emerge in the spectrum at the kinetic positron energy $E_{e^{+}}=\left|E_{1 s \sigma}^{B}\left(R_{\min }\right)\right|-2 m c^{2}$, i.e., at the position of the resonant $1 s \sigma$ state below the threshold of the negative-energy continuum. ${ }^{9,10}$ A small fraction of delayed reactions would suffice to produce visible structures in the resulting spectrum. ${ }^{10}$ While the "EPOS" and "ORANGE" experimental groups at GSI indeed have detected narrow peaks in positron spectra from heavy-ion collisions, ${ }^{5,15}$ the interpretation of these structures as spontaneous positron emission appears to have failed (cf. the contributions in Ref. 16). At present there is no convincing evidence for the presence of the required long delay times. However, alternative attempts to explain the positron peaks also meet severe difficulties. Therefore, in Sec. III B we study the modification expected for bare nucleus experiments also for this hypothetical model to see whether more light can be thrown on the question of the positron lines by such means. Finally, in Sec. V a brief summary will be given.

\section{THEORETICAL FRAMEWORK}

The semiclassical approximation is used to describe the dynamics of electrons and positrons during a heavy-ion collision. Our calculation is based on the time-dependent Dirac equation

$i \hbar \frac{\partial}{\partial t} \Phi_{i}(\mathbf{r}, t)=\left\{c \alpha \cdot \mathbf{p}+\beta m c^{2}+[V(\mathbf{r}, \mathbf{R}(t))]_{l=0}\right\} \Phi_{i}(\mathbf{r}, t)$,

with the two-center potential $V(r, R(t))$ in monopole approximation $(l=0)$. $\mathbf{R}(t)$ is the time-dependent separation between the two nuclear centers. Equation (1) determines the motion of a single electron, initially in state $i$, in the external time-varying electromagnetic field of the two nuclei. At nonrelativistic bombarding energies it is appropriate to expand the wave functions $\Phi_{i}(r, t)$ in a complete basis $\phi_{k}(\mathbf{r}, R)$ of adiabatic, molecular wave functions of the Dirac Hamiltonian. They are chosen to satisfy

$\left\{c \boldsymbol{\alpha} \cdot \mathbf{p}+\beta m c^{2}+[V(\mathbf{r}, \mathbf{R})]_{l=0}\right\} \phi_{k}(\mathbf{r}, R)=E_{k}(R) \phi_{k}(\mathbf{r}, R)$.

In the case of fixed-target-scattering experiments we must account for screening due to target and projectile electrons. In the adiabatic Hartree-Fock-Slater (HFS) approximation, ${ }^{17}$ the two-center potential is taken as

$$
V(\mathbf{r}, \mathbf{R})=\left[V_{\mathrm{Cb}}(\mathbf{r}, \mathbf{R})+V_{\mathrm{HFS}}(\mathbf{r}, \mathbf{R})\right]_{l=0},
$$

where $V_{\mathrm{Cb}}$ is the Coulomb potential of the nuclei and $V_{\text {HFS }}$ is the self-consistent potential generated by the adiabatic many-electron state. For crossed-beam experiments with bare uranium nuclei, where no electrons are present initially, a basis $\phi_{k}^{\mathrm{Cb}}(\mathbf{r}, R)$ of unscreened Coulombic states must be taken.

Inserting the expansion of the time-dependent wave function $\Phi_{i}(\mathbf{r}, t)$,

$$
\Phi_{i}(\mathbf{r}, t)=\sum_{k} a_{i k}(t) \phi_{k}(\mathbf{r}, R(t)) e^{-i \chi_{k}(t)},
$$

into (1) and projecting with the corresponding states $\phi_{j}(\mathbf{r}, R)$, we are led to a set of first-order coupled differential equations for the occupation amplitudes $a_{i k}(t)$,

$$
\begin{array}{r}
\dot{a}_{i j}(t)=-\sum_{k(\neq j)} a_{i k}(t)\left\langle\phi_{j}|\partial / \partial t| \phi_{k}\right\rangle \\
\times \exp \left[i\left(\chi_{j}-\chi_{k}\right)\right],
\end{array}
$$

with the phases

$$
\chi_{k}(t)=\frac{1}{\hbar} \int^{t} E_{k}\left(R\left(t^{\prime}\right)\right) d t^{\prime}
$$

Neglecting electron correlations, the probability that a particle occupies a state $p$ above the Fermi level $F$ after the collision reads

$$
N_{p}=2 \sum_{k<F}\left|a_{k p}(+\infty)\right|^{2}(p>F),
$$

while the probability of formation of a hole in a state $q$ below the Fermi level $F$ is

$$
N_{q}=2 \sum_{k>F}\left|a_{k q}(+\infty)\right|^{2} \quad(q<F) .
$$

In accordance with standard terminology, positrons count as holes in the negative-energy continuum. Equations (7) and (8) contain a summation over spin orientations. Only the dominant channels with $j=\frac{1}{2}$ will be taken into account. The position of the Fermi level $F$ is determined by the initial conditions. Throughout we will take $F=\left(3 s \sigma, 4 p_{1 / 2} \sigma\right)$ for fixed-target experiments. The scattering of bare nuclei in cross-beam collisions will be denoted by the Fermi level $F=0$.

The description of positron production in supercritical collisions systems, where $Z_{P}+Z_{T}$ exceeds $Z_{\text {cr }}$, requires a 
modification of this formalism which was first presented in Ref. 8. In this description, atomic positron creation in supercritical heavy-ion collisions is found to originate from two processes which are "anschaulich" and formally well distinguished, but act coherently.

Firstly, the dynamical positron emission process which is proportional to the radial velocity $\dot{R}$ of the two nuclear centers and to the $R$-dependent radial matrix elements.

Secondly, spontaneous positron creation, where only the $R$-dependent spontaneous matrix elements enter. It does not require nuclear motion and leads, in the static limit $R(t)=$ const $<R_{\text {cr }}$, to the exponential decay of a vacancy prepared in the quasibound $1 s \sigma$ state. The decay width $\Gamma_{R}$ is typically of the order of a few keV.

To summarize, for the conventional fixed-targetscattering experiment as well as for crossed beams of bare nuclei we apply the formalism sketched above to calculate atomic excitation processes. However, there are two basic differences, the consequences of which are easy to predict qualitatively.

(a) The change of the Fermi surface from $F=\left(3 s \sigma, 4 p_{1 / 2} \sigma\right)$ to $F=0$ will make a substantial difference due to the removal of Pauli blocking. In order to calculate, e.g., the creation probability for a positron of given energy, one has to sum the transition probabilities into all bound states and into the upper electron continuum, whereas only the continuum and some high-lying bound states contribute in fixed-target scattering. Transitions from the negative-energy continuum into the vacant $1 s \sigma$ state, usually suppressed by the small probability for creating a $1 s \sigma$ hole during nuclear approach, now have a chance to dominate the positron spectra. ${ }^{8}$ Thus we expect the spontaneous, as well as the dynamical, positron production to be enhanced drastically.

(b) A second, slightly more subtle distinction between the two types of experiments lies in the absence of electron screening. For ordinary fixed-target scattering, the time-dependent atomic wave function $\Phi_{i}(R(t))$ is expanded in a basis $\phi_{k}^{\mathrm{HFS}}(\mathrm{r}, R)$ of self-consistent adiabatic quasimolecular wave functions including electron screening in the Hartree-Fock-Slater approximation. For these calculations it is assumed that only 50 electrons in the highest molecular states are missing. ${ }^{17}$ In contrast, in collisions of bare nuclei, no electron is initially present, thus requiring an expansion of $\Phi_{i}$ in quasimolecular Coulombic wave functions $\phi_{k}^{\mathrm{Cb}}(\mathrm{r}, R)$. This will lead ${ }^{17}$ to a deeper binding of electron states.

In particular, the resonant $1 s \sigma$ state "dives" more deeply into the negative energy continuum and, at the same time, obtains an increased resonance decay width $\Gamma_{R}$. Typical values for the binding energy $E_{1 s \sigma}^{B}$ and the resonance with $\Gamma_{R}$ of the supercritical $1 s$ state in a $\mathrm{U}+\mathrm{U}$ quasiatom are given in Table $I$. The absence of screening in the fully ionized system increases the binding energy by about $100 \mathrm{keV}$. At the same time we notice an increase of the spontaneous decay width $\Gamma_{R}$ by a factor of about 3.5 for $R=18 \mathrm{fm}$ and of about 2.7 for $R=16.5 \mathrm{fm}$, respectively, when switching off the electron-electron interaction. Thus the spontaneous positron creation proba-
TABLE I. Binding energy and spontaneous decay width of the resonant $1 s \sigma$ electron state for two internuclear distances $R$ in the $\mathrm{U}+\mathrm{U}$ system. The entries HFS and $\mathrm{Cb}$ refer to electron states including electron shielding in a 50-fold ionized system or to unscreened Coulombic electron states, respectively. $E_{\text {lab }}$ is the impact energy leading to the distance of closest approach $R$ for central collisions, neglecting nuclear interaction.

\begin{tabular}{cccccc}
\hline \hline & & \multicolumn{2}{c}{$E_{\text {lso }}^{B}(\mathrm{keV})$} & \multicolumn{2}{c}{$\Gamma_{R}(\mathrm{keV})$} \\
$R(\mathrm{fm})$ & $E_{\mathrm{lab}}(\mathrm{MeV} / \mathrm{u})$ & HFS & $\mathrm{Cb}$ & HFS & $\mathrm{Cb}$ \\
\hline 18.0 & 5.7 & 1165 & 1270 & 0.31 & 1.07 \\
16.5 & 6.2 & 1200 & 1310 & 0.63 & 1.69 \\
\hline \hline
\end{tabular}

bility per existing $K$ vacancy will be notably enhanced in bare collisions. For very long nuclear reaction times, this yields a positron line shifted by about $100 \mathrm{keV}$.

In the following we want to discuss these effects more quantitatively, namely, for the case of $U+U$ scattering at two center-of-mass kinetic energies of the colliding nuclei, $E_{\text {rel }}=680$ and $740 \mathrm{MeV}$. These energies correspond to $E_{\text {lab }}=5.7$ and $6.2 \mathrm{MeV} / \mathrm{u}$, respectively, when bombarding stationary uranium targets with uranium ions. In crossed-beam collisions, the relative angle between the uranium beams and the kinetic energy of the high-energy particles have to be adjusted such as to obtain the desired kinematical conditions. For eight impact parameters ( $b=0$ up to $b=40 \mathrm{fm}$ ), transitions between more than 6 bound states, 15 upper continuum, and 30 lower continuum states, both for the $s_{1 / 2}$ and $p_{1 / 2}$ angular momentum channels $(\kappa=+1$ and $\kappa=-1)$, have been calculated.

\section{QUANTITATIVE DIFFERENCES IN POSITRON CREATION}

\section{A. Rutherford scattering}

We first want to discuss the positron yields for elastic $\mathrm{U}+\mathrm{U}$ scattering assuming that the nuclei move along ordinary Rutherford trajectories. Figure 1 shows the positron spectra at $E_{\text {rel }}=740 \mathrm{MeV}$ for various impact parameters between 0 and $40 \mathrm{fm}$. For head-on collisions, $b=0$, the maximum differential emission probability $d P^{e^{+}} /\left.d E_{e^{+}}\right|_{\max }$ will be enhanced by a factor of about 30 , namely, from $6.2 \times 10^{-7} \mathrm{keV}^{-1}$ for conventional scattering [Fig. 1(a)] up to $1.76 \times 10^{-5} \mathrm{keV}^{-1}$ for bare nuclei [Fig. 1(b)]. To explain this large increase it is important to remember that in the collision of bare nuclei, two vacancies, due to spin degeneracy, are already present in the quasimolecular $1 s \sigma$ state, while in fixed-target scattering the probability for dynamically produced $1 s \sigma$ holes at the distance of closest approach $(R=2 a)$ is only about 0.055 (normalized to 2). Thus the maximum differential emission probability in the $s_{1 / 2}$ channel rises drastically by more than one of magnitude. For the $p_{1 / 2}$ channel the effect is smaller (due to the larger $2 p_{1 / 2} \sigma$ hole probability in conventional scattering): its contribution increase by a factor of about 10 . For the lower bombarding energy of $E_{\text {rel }}=680 \mathrm{MeV}$ we find an even stronger increase in the positron spectra by a factor of about 40 , namely, from $4 \times 10^{-7}$ to $1.6 \times 10^{-5} \mathrm{keV}^{-1}$. 
In more distant collisions, here for $740 \mathrm{MeV}$ relative kinetic energy, the enhancement factor increases from about 30 in central collisions (as discussed for $b=0$ ) to about 65 in medium-impact parameter collisions $(b \simeq 25$ fm) and up to a factor of about 130 in peripheral collisions at $b \simeq 40 \mathrm{fm}$. This effect again is caused by the strong suppression of positron creation in conventional peripheral scattering due to the rapidly falling $K$-vacancy probabilities with $b$. For all impact parameters calculated, the position of the maximum in the energy distribution of induced positrons is shifted towards higher kinetic positron energies. Since the binding energies are considerably larger in collisions of bare nuclei due to the absence of screening as discussed above, the corresponding positron spectra exhibit a shift of about $40-50 \mathrm{keV}$ towards higher kinetic positron energies.

All features discussed so far are revealed also in the spectra of emitted positrons originating from Rutherford scattering of $U+U$ in finite angular windows: the increase in probability, being most pronounced for distant collisions, the energetic shift of the spectral maximum towards higher kinetic energies, and furthermore (not visible in a linear plot like Fig. 1), a steeper decrease in the high-energy tail of the spectrum.

Pair creation with the electron ending up in the $K$ shell is the dominant effect in the scattering of bare nuclei (see also discussion in Sec. IV). However, not only the dynamically induced part of the positron spectrum is enhanced, but also the contribution from spontaneous positron creation is enhanced. This will become more transparent in Sec. III B.

\section{B. Positron production in heavy-ion collisions with time delay}

In Sec. II we have pointed out that spontaneous positron creation can become dominant only in heavy-ion
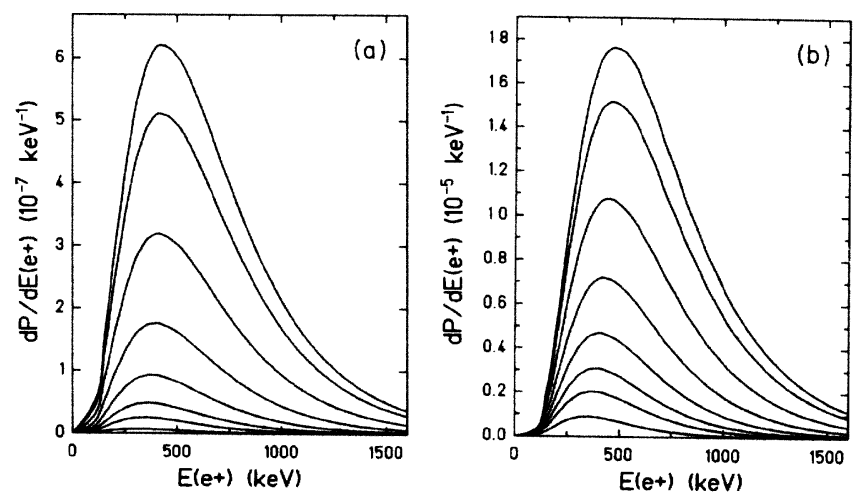

FIG. 1. Energy distribution of positrons emitted in $U+U$ collisions at a center-of-mass kinetic energy of $E_{\text {rel }}=740 \mathrm{MeV}$ for impact parameters $b=0$ (upper curve) to $b=40 \mathrm{fm}$. The considered impact parameters are $0,5,10,15,20,25,30$, and 40 $\mathrm{fm}$, respectively. Comparing conventional scattering (a) to bare nuclei scattering (b), enhancement factors between about 30 for head-on collisions and 130 for distant collisions are found. In (b), an energy shift towards higher kinetic positron energies of about $50 \mathrm{keV}$ (for $b=0$ ) is noticed. The steeper decrease of the spectra in the high-energy region is not visible on this linear plot. collisions with time delay. Since we want to investigate this contribution, we have applied the schematic trajectory model for the nuclear motion during a prolonged reaction as described in Refs. 10 and 12. The two nuclei approach each other on a hyperbolic Rutherford trajectory prescribed by the initial bombarding energy $E_{\text {lab }}$ and the impact parameter $b$. At the distance of closest approach $R_{\text {min }}$ or some other characteristic distance $R_{0}$ they stay together for a period of time $T$, which can be varied as a free parameter. After this time interval has elapsed the two nuclei separate and escape to infinity on another Rutherford half-trajectory, where we may allow for angular momentum and energy dissipation during the nuclear contact.

Positron spectra $d P / d E_{e^{+}}$for pure Rutherford scattering, i.e., $T=0$, and for various values of the nuclear delay time in the interval $T=10^{-21}$ to $10^{-20} \mathrm{~s}$ are displayed in Fig. 2. The results refer to head-on $U+U$ collisions at a center-of-mass kinetic energy $E_{\mathrm{rel}}=740 \mathrm{MeV}$. In both cases, conventional scattering [Fig. 2(a)] and bare nuclei [Fig. 2(b)], excitation rates increase monotonically with growing delay time $T$. There are quantitative differences, however, since in the latter case the availability of $K$ vacancies favors the spontaneous $e^{+}$creation mechanism leading to a more pronounced increase with $T$. This effect is particularly visible for small delay times, where the rates in ordinary collisions remain nearly constant while bare collisions already lead to a significant increase. For example, for the time $T=1 \times 10^{-21} \mathrm{~s}$, a value which can be realistically attained in deep-inelastic collisions, ${ }^{14,18}$ the respective enhancement factors are 1.8 versus 1.18. At the hypothetical larger value $T=1 \times 10^{-20}$ s of the delay time, the factors are 15 and 7.

For asymptotically long nuclear reaction times $T \gg \hbar / \Gamma_{R}$, the total emission probability in the spontaneous line will be 0.055 and about 2 per collision, according to the available number of holes in the $1 s \sigma$ state.
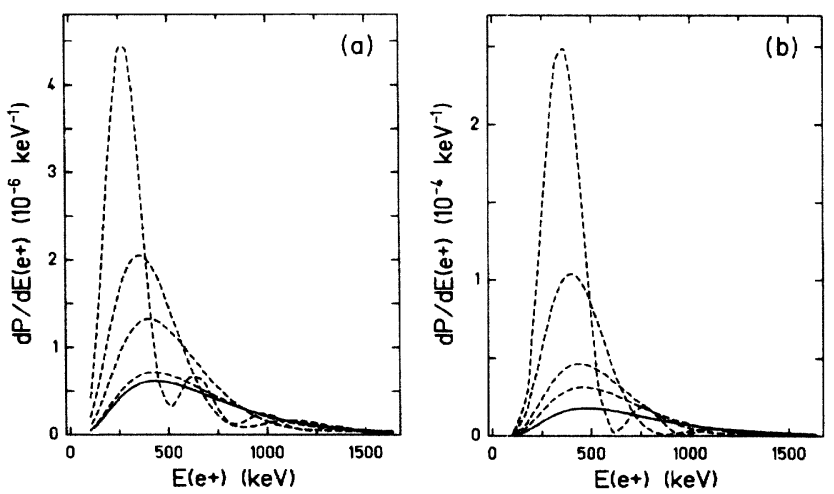

FIG. 2. Differential probability for positron emission in $E_{\text {rel }}=740 \mathrm{MeV} \mathrm{U}+\mathrm{U}$ head-on collisions for pure Rutherford scattering ( $T=0$, solid line) and nuclear time delays of $T=1,2$, 5 , and $10 \times 10^{-21} \mathrm{~s}$ (dashed lines). For very large nuclearreaction times $T \gg \hbar \Gamma_{R}$, the total positron probabilities would be about 0.055 (a) and about 2 (b), respectively, reflecting the $K$-hole probabilities at the distance of closest approach, $P_{1 s \sigma}$ $(t=0)$. 
For higher center-of-mass collision energies, the increase in the maximum differential emission probability as well as in the total positron probability will be smaller since here the $1 s \sigma$ hole probability $P_{1 s \sigma}\left(R_{\min }=2 a\right)$ is larger already in conventional scattering. The opposite holds true for lower bombarding energies.

A more realistic model for the nuclear reaction process has to account for the fact that the nuclear delay time will follow a distribution function instead of having a fixed value $T{ }^{19,20}$ As an example, Fig. 3 shows the resulting positron spectra calculated with an exponential time distribution

$$
\left\langle\frac{d P}{d E_{e^{+}}}\right\rangle=\frac{1}{\tau} \int_{0}^{\infty} d T e^{-T / \tau} \frac{d P}{d E_{e^{+}}}(T) .
$$

Mean time values of $\tau=1$ to $4 \times 10^{-21} \mathrm{~s}$ have been used. The qualitative behavior is similar to that found for fixed delay times $T$.

The spectra displayed in Figs. 2 and 3 also reveal the energetic shift of the resonant $1 s \sigma$ state within the lower electron continuum due to the absence of electron screening. With increasing nuclear delay time $T$, the position of the spontaneous positron line shifts from the dynamically caused probability maximum $\left.E_{e^{+}}\right|_{\max } \simeq 400$ and 450 $\mathrm{keV}$, respectively, to the position of the resonant state, i.e., $E_{e^{+}}=E_{1 s \sigma}^{B}\left(R_{0}\right)-2 m c^{2}$, cf. Table I. In the asymptotic limit this would yield maxima at 140 and $250 \mathrm{keV}$, respectively, for $E_{\text {rel }}=680 \mathrm{MeV}$, and 180 and $290 \mathrm{keV}$, respectively, for $E_{\mathrm{rel}}=740 \mathrm{MeV}$. The observation of this charge-state dependence of the positron line structure would constitute a direct proof that they are of atomic origin.

Finally, let us review the attempts to explain the positron line structures observed at GSI (ref. 5) within the framework of spontaneous positron production. In Refs. 10 it was demonstrated how a small fraction $q \lesssim 10^{-2}$ of nuclear reactions with very long delay times $T \gtrsim 3 \times 10^{-20} \mathrm{~s}$ can produce a narrow-peak structure resembling the positron spectra measured in $U+U$ and $\mathrm{U}+\mathrm{Cm}$ collisions. Meanwhile, additional line structures have been found which appear also in subcritical col-
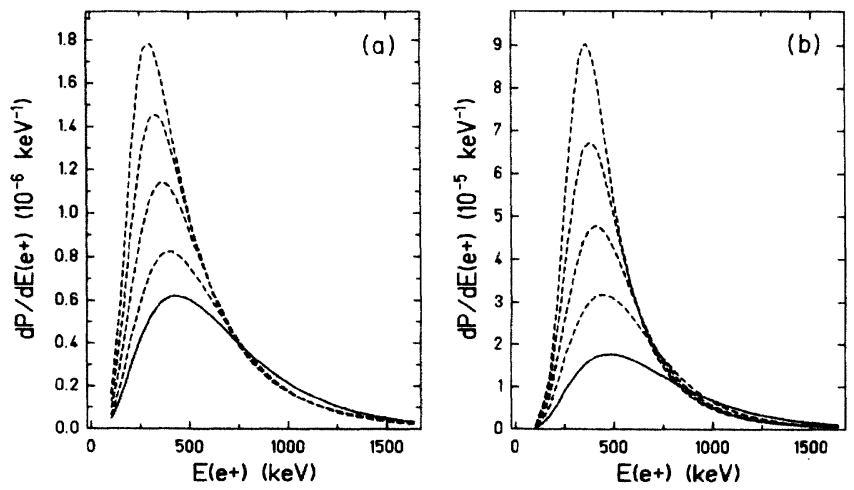

FIG. 3. Same as Fig. 2, assuming an exponential distribution of delay times instead of a fixed value $T$. The values of the mean delay time have been chosen as $\tau=1,2,3$, and $4 \times 10^{-21} \mathrm{~s}$ (dashed lines). lisions and which do not conform with the energy systematics expected for spontaneous positron production. Furthermore, correlated $e^{+} e^{-}$events have been discovered. ${ }^{15}$ While there are many speculative attempts to interpret the data, at present the question has to be considered open, cf. Ref. 16.

Nuclear-physics calculations on the interaction of very heavy nuclei at energies very close to the Coulomb barrier are highly model dependent. The existence of "pockets" in the internuclear potential ${ }^{21}$ has been discussed and collisions with sticking times longer than those known from deep-inelastic collisions are not ruled out. In principle, some of the observed line structures still may be related to such an effect. Therefore it is not without interest to study how this mechanism would be modified in collisions of bare nuclei.

As an illustrative example, Fig. 4 shows a hypothetical superposition of a positron spectrum originating from Rutherford scattering of $\mathrm{U}+\mathrm{U}$ at $E_{\mathrm{rel}}=740 \mathrm{MeV}$ in an angular window of $\theta_{\mathrm{cm}}=90^{\circ} \pm 6^{\circ}$ and the spectrum of spontaneous positrons from nuclear molecule formation of sharp lifetime $T=5 \times 10^{-20} \mathrm{~s}$ with a nuclear-reaction cross section $\sigma_{N}(\theta) \simeq 7 \mathrm{mb}$ in the assumed angular window. (Note the difference to Figs. 2 and 3 where no superposition of delayed and undelayed collisions was assumed.) In conventional scattering experiments, the spontaneous positron line is not very pronounced. In contrast, for bare nuclei, spontaneous positrons give a much more prominent contribution in the energy range $E_{e^{+}} \simeq 250-350 \mathrm{keV}$. For $E_{\text {rel }}=680 \mathrm{MeV}$, however, the calculation yielded less conclusive results. Remember also that the values for the nuclear-reaction cross section $\sigma_{N}(\theta)$ and for the sharp delay time $T$ as well as the nuclear charge distribution during the reaction (two spheres
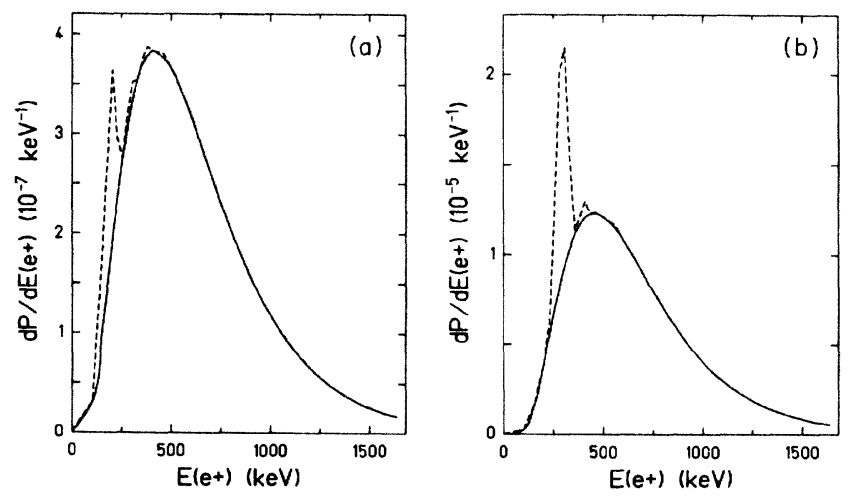

FIG. 4. Spectra of positrons stemming from Rutherford scattering of $U+U$ at $E_{\text {rel }}=740 \mathrm{MeV}$ in a window of centerof-mass scattering angles of $\theta_{\mathrm{cm}}=90^{\circ} \pm 6^{\circ}$ superposed by an additional contribution originating from hypothetical long-lasting nuclear reactions with delay time $T=5 \times 10^{-20} \mathrm{~s}$ and nuclear cross section $\sigma_{N}(\theta) \simeq 7 \mathrm{mb}$ (dashed line). Pure Rutherford scattering is indicated by the solid line. While for conventional scattering experiments (a) the spontaneous positron line would be hard to detect, in bare nuclei collisions (b) a line structure at the higher energy $E_{e^{+}}=285 \mathrm{keV}$ (for the kinematical assumptions made) is dominating the positron spectrum. 
TABLE II. Results of coupled-channel calculations for $U+U$ collisions at center-of-mass kinetic energies 740 and $680 \mathrm{MeV}$. Column 3, the impact-parameter dependent probabilities $P_{e^{+}}^{\text {bound }}$ to excite electrons from the negative energy continuum into empty bound $s$ states and $p_{1 / 2}$ states vs impact parameter $b$. Columns 4 and 5 , the total positron production probabilities $\boldsymbol{P}_{e^{+}}$for two different choices of the Fermi surface. Almost all electrons from pair production finally occupy bound states.

\begin{tabular}{ccccc}
\hline \hline$E_{\text {rel }}(\mathrm{MeV})$ & $b(\mathrm{fm})$ & $P_{e^{+}}^{\text {bound }}(F=0)$ & $P_{e^{+}}(F=0)$ & $P_{e^{+}}\left(F=\left(3 s_{1 / 2} \sigma, 4 p_{1 / 2} \sigma\right)\right)$ \\
\hline 6.2 & 0 & $1.23 \times 10^{-2}$ & $1.26 \times 10^{-2}$ & $4.83 \times 10^{-4}$ \\
& 5 & $1.04 \times 10^{-2}$ & $1.06 \times 10^{-2}$ & $3.91 \times 10^{-4}$ \\
& 10 & $7.04 \times 10^{-3}$ & $7.15 \times 10^{-3}$ & $2.32 \times 10^{-4}$ \\
& 15 & $4.41 \times 10^{-3}$ & $4.47 \times 10^{-3}$ & $1.20 \times 10^{-4}$ \\
& 20 & $2.71 \times 10^{-3}$ & $2.73 \times 10^{-3}$ & $5.84 \times 10^{-5}$ \\
& 25 & $1.67 \times 10^{-3}$ & $1.68 \times 10^{-3}$ & $2.80 \times 10^{-5}$ \\
& 30 & $1.04 \times 10^{-3}$ & $1.04 \times 10^{-3}$ & $1.34 \times 10^{-5}$ \\
& 40 & $4.11 \times 10^{-4}$ & $4.11 \times 10^{-4}$ & $3.15 \times 10^{-6}$ \\
& & & \\
5.7 & 0 & $1.04 \times 10^{-2}$ & $1.06 \times 10^{-2}$ & $2.93 \times 10^{-4}$ \\
& 5 & $8.86 \times 10^{-3}$ & $8.97 \times 10^{-3}$ & $2.40 \times 10^{-4}$ \\
& 10 & $6.05 \times 10^{-3}$ & $6.12 \times 10^{-3}$ & $1.45 \times 10^{-4}$ \\
15 & $3.80 \times 10^{-3}$ & $3.83 \times 10^{-3}$ & $7.50 \times 10^{-5}$ \\
& 20 & $2.33 \times 10^{-3}$ & $2.34 \times 10^{-3}$ & $3.65 \times 10^{-5}$ \\
& 25 & $1.43 \times 10^{-3}$ & $1.43 \times 10^{-3}$ & $1.74 \times 10^{-5}$ \\
& 30 & $8.80 \times 10^{-4}$ & $8.80 \times 10^{-4}$ & $8.24 \times 10^{-5}$ \\
& 40 & $3.42 \times 10^{-4}$ & $3.42 \times 10^{-4}$ & $1.88 \times 10^{-6}$ \\
\hline \hline
\end{tabular}

with internuclear separation $R=2 a$ ) have been chosen quite arbitrarily so that no quantitative predictions are intended. Nevertheless one can note that the scattering of bare nuclei would allow to conclude definitely whether (some of) the observed positron lines are related to the $K$ shell of the united atom. A drastic increase in intensity and, perhaps even more significantly, an energy shift would signal such an origin.

\section{THE FILLING OF EMPTY BOUND STATES}

In Sec. III we have shown that the existence of empty inner-shell electron states in scattering of bare uranium ions amplifies the positron creation processes. This is due to the fact that they serve as dominant final states for electrons from pair creation. In Table II, column 3, we have summarized the impact-parameter-dependent probabilities $P_{e^{+}}^{\text {bound }}$ to excite an electron from the negativeenergy continuum into an empty bound state for the two relative kinetic energies $E_{\text {rel }}=740$ and $680 \mathrm{MeV}$. Impact parameters $b=0$ up to $b=40 \mathrm{fm}$ have been chosen. The probability to excite into an $s$ state is larger by factors between 6 and 20 compared to the corresponding probability for excitation into a $p_{1 / 2}$ state. The sum of both angular momentum contributions (column 3 ) is nearly the same as the total positron production probability $\boldsymbol{P}_{e^{+}}$ (column 4), the latter being defined as the energy integral of the differential positron production probability. That is, almost all electrons from dynamical and spontaneous pair-production processes are captured into inner-shell bound states.

The table also shows the influence of the Pauli blocking on the total positron probabilities. Switching from $F=0$ (column 4) to $F=\left(3 s_{1 / 2} \sigma, 4 p_{1 / 2} \sigma\right)$ (column 5) for
$E_{\text {rel }}=740 \mathrm{MeV}$ we find a decrease by a factor of about 25 for $b=0$ and of about 130 for $b \simeq 40 \mathrm{fm}$, a result already discussed in Sec. III.

The probabilities of electron capture from the vacuum are easily measured independently by detecting the charge change of the beam, provided that electroncapture processes from the rest gas in the ion accelerator can be neglected. The total capture cross section $\sigma_{e^{+}}^{\text {bound, }}$ obtained by integrating the corresponding probabilities over impact parameter, amounts to 125 and $105 \mathrm{mb}$ for the two collision energies. In collisions with filled inner shells, the integrated positron creation cross section is only 2.8 or $2.3 \mathrm{mb}$, respectively. Note that our values $P_{e^{+}}^{\text {bound }}$ and $\sigma_{e^{+}}^{\text {bound }}$ are inclusive quantities, encompassing single-electron and multielectron capture. However, in view of the small absolute values of the capture probabilities, single-electron final states will make the dominant contribution in the present case.

\section{CONCLUSIONS}

We have studied positron creation in collisions of crossed beams of two bare uranium nuclei, contrasting the fixed-target-scattering experiments with highly stripped ion collisions carried out at present. Calculating positron spectra within the coupled-channel formalism we have shown that the absence of electron screening causes a $\sim 50-\mathrm{keV}$ shift of the spectral maximum towards higher kinetic energies in pure Rutherford scattering and $a \gtrsim 100-\mathrm{keV}$ shift in time-delayed heavy-ion scattering the exact values depending on the nuclear dynamics.

Furthermore, since transitions from the negativeenergy continuum into the vacant bound states, especially the two $1 s \sigma$ states, are no longer suppressed by the small 
probabilities for having created a $1 s \sigma$ hole, positron production probabilities increase by a factor of $\sim 30$, e.g., in $740-\mathrm{MeV}$ central collisions and by up to a factor of $\sim 130$ in distant $(b \simeq \mathbf{4 0} \mathrm{fm})$ collisions assuming pure Rutherford scattering. In this way we have shown that innershell states, if brought empty into the collision, will represent the dominant final states for electrons from pair production.

Using a schematic model we also have studied collisions with time delays resulting from nuclear scattering. In fully ionized collisions, positron production is dominated by the contribution from the $1 s$ state. Since this contribution in supercritical collisions systems is sensitive to the duration of the period of supercriticality, an increase in intensity will be visible already at modest values of the delay time. This was demonstrated for the value $T \sim 10^{-21} \mathrm{~s}$ which can be achieved in deep-inelastic collisions.

Hypothetical collisions with very long delay times would produce narrow positron lines due to the spon- taneous decay mechanism. Whether some of the observed positron lines ${ }^{5}$ are related to this mechanism could be decided by using fully ionized collisions. A strong rise in intensity and a shift in line energy to higher values are predicted.

Putting aside possible experimental difficulties, the scattering of bare nuclei in crossed-beam experiments thus seems to be encouraging tool to yield additional information on inner shells in superheavy atomic systems. We finally note that part of the enhancement of positron production discussed in this paper can be achieved already in collisions with a fully ionized projectile impinging on a stationary neutral target atom since here, on the average, one hole is transferred to the quasimolecular $1 s \sigma$ state.

\section{ACKNOWLEDGMENT}

We thank Professor Walter Meyerhof for helpful criticism of the manuscript.
*Present address: Abteilung TEB, Hoechst AG., Postfach 800320 , D-6230 Frankfurt am Main 80, Federal Republic of Germany.

${ }^{1}$ W. Greiner, B. Müller, and J. Rafelski, Quantum Electrodynamics of Strong Fields (Springer, Berlin, 1985).

${ }^{2}$ P. H. Mokler and D. Liesen, in Progress in Atomic Spectroscopy, Part C, edited by H. J. Beyer and H. Kleinpoppen (Plenum, New York, 1984).

${ }^{3}$ M. A. Herath Banda, W. Koenig, B. Martin, F. Güttner, H. Skapa, J. Soltani, K. Dworschak, F. Bosch, Ch. Kozhuharov, and A. V. Ramayya, Phys. Rev. A 33, 861 (1986).

${ }^{4}$ H. Backe, L. Handschug, F. Hessberger, E. Kankeleit, L. Richter, F. Weik, R. Willwater, H. Bokemeyer, P. Vincent, Y. Nakayama, and J. S. Greenberg, Phys. Rev. Lett. 40, 1443 (1978); C. Kozhuharov, P. Kienle, E. Berdermann, H. Bokemeyer, J.S. Greenberg, Y. Nakayama, P. Vincent, H. Backe, L. Handschug, and E. Kankeleit, ibid. 42, 376 (1979).

${ }^{5} \mathrm{~J}$. Schweppe, A. Gruppe, K. Bethge, H. Bokemeyer, T. Cowan, H. Folger, J. S. Greenberg, H. Grein, S. Ito, R. Schulé, D. Schwalm, K. E. Stiebing, N. Trautmann, P. Vincent, and M. Waldschmidt, Phys. Rev. Lett. 51, 2261 (1983); M. Clemente, E. Berdermann, P. Kienle, H. Tsertos, W. Wagner, C. Kozhuharov, F. Bosch, and W. Koenig, Phys. Lett. 137B, 41 (1984); T. Cowan, H. Backe, M. Begemann, K. Bethge, H. Bokemeyer, H. Folger, J. S. Greenberg, H. Grein, A. Gruppe, Y. Kido, M. Klüver, D. Schwalm, J. Schweppe, K. E. Stiebing, N. Trautmann, and P. Vincent, Phys. Rev. Lett. 54, 1761 (1985); H. Tsertos, E. Berdermann, F. Bosch, M. Clemente, P. Kienle, W. Koenig, C. Kozhuharov, and W. Wagner, Phys. Lett. 162B, 273 (1985); P. Kienle, J. Phys. Soc. Jpn. Suppl. II 54, 549 (1985).

${ }^{6}$ W. Betz, G. Heiligenthal, J. Reinhardt, R. K. Smith, and W. Greiner, in Proceedings of the Ninth International Conference on the Physics of Electronic and Atomic Collisions, Seattle, 1975 , edited by J. S. Risley and R. Geballe (University of Washington Press, Seattle, 1976), p. 531.

${ }^{7}$ P. Kienle (unpublished).

8J. Reinhardt, B. Müller, and W. Greiner, Phys. Rev. A 24, 103 (1981).

9J. Rafelski, B. Müller, and W. Greiner, Z. Phys. A 285, 49
(1978).

10J. Reinhardt, U. Müller, B. Müller, and W. Greiner, Z. Phys. A 303, 173 (1981); U. Müller, G. Soff, T. de Reus, J. Reinhardt, B. Müller, and W. Greiner, ibid. 313, 263 (1983); U. Müller, T. de Reus, J. Reinhardt, B. Müller, and W. Greiner, ibid. 323, 261 (1986).

${ }^{11} \mathrm{~A}$ quantum-mechanical treatment of nuclear scattering and atomic excitation has been carried out in U. Heinz, J. Reinhardt, B. Müller, W. Greiner, and U. Müller, Z. Phys. A 314, 125 (1983); U. Heinz, U. Müller, J. Reinhardt, B. Müller, and W. Greiner, Ann. Phys. (N.Y.) 158, 476 (1984).

${ }^{12}$ G. Soff, J. Reinhardt, B. Müller, and W. Greiner, Phys. Rev. Lett. 43, 1981 (1979).

${ }^{13}$ U. Müller, G. Soff, J. Reinhardt, T. de Reus, B. Müller, and W. Greiner, Phys. Rev. C 30, 1199 (1984).

${ }^{14}$ H. Backe, P. Senger, W. Bonin, E. Kankeleit, M. Krämer, R. Krieg, V. Metag, N. Trautmann, and J. B. Wilhelmy, Phys. Rev. Lett. 50, 1838 (1983); R. Krieg, E. Bozek, U. Gollerthan, E. Kankeleit, G. Klotz-Engmann, M. Krämer, U. Meyer, H. Oeschler, and P. Senger, Phys. Rev. C 34, 562 (1986).

${ }^{15}$ T. Cowan, H. Backe, K. Bethge, H. Bokemeyer, H. Folger, J. S. Greenberg, K. Sakaguchi, D. Schwalm, J. Schweppe, K. E. Stiebing, and P. Vincent, Phys. Rev. Lett. 56, 444 (1986).

${ }^{16}$ Physics of Strong Fields, Vol. 153 of NATO Advanced Study Institute Series B: Physics, edited by W. Greiner (Plenum, New York, 1987).

${ }^{17}$ T. de Reus, J. Reinhardt, B. Müller, W. Greiner, G. Soff, and U. Müller, J. Phys. B 17, 615 (1984); T. de Reus, GSI Report No. GSI-87-8, ISSN 0171-4546.

${ }^{18} \mathrm{~W}$. Meyerhof and J.F. Chemin, in Advances in Atomic and Molecular Physics, edited by D. R. Bates and B. Bederson (Academic, New York, 1984), Vol. 20.

${ }^{19}$ J. Reinhardt, B. Müller, W. Greiner, and U. Müller, Phys. Rev. A 28, 2558 (1983).

${ }^{20}$ T. Tomoda and H. A. Weidenmüller, Phys. Rev. C 83, 739 (1983).

${ }^{21}$ M. Seiwert, W. Greiner, and W. T. Pinkston, J. Phys. G 11, L21 (1985); A. Faessler, M. Ismail, N. Ohtsuka, M. Rashadan, and W. Wadia, Z. Phys. A 326, 501 (1987). 